\title{
Does Blue Light Restore Human Epidermal Barrier Function via Activation of Opsin During Cutaneous Wound Healing?
}

\author{
Irene Castellano-Pellicena, ${ }^{1,2}$ Natallia E. Uzunbajakava, ${ }^{2}$ Charles Mignon, ${ }^{1,2}$ Bianca Raafs, ${ }^{2}$ \\ Vladimir A. Botchkarev, ${ }^{1}$ and M. Julie Thornton ${ }^{1 *}$ \\ ${ }^{1}$ Centre for Skin Sciences, Faculty of Life Sciences, University of Bradford, Bradford, United Kingdom \\ ${ }^{2}$ Philips Research, High Tech Campus, Eindhoven, The Netherlands
}

Background and Objective: Visible light has beneficial effects on cutaneous wound healing, but the role of potential photoreceptors in human skin is unknown. In addition, inconsistency in the parameters of blue and red light-based therapies for skin conditions makes interpretation difficult. Red light can activate cytochrome $\mathrm{c}$ oxidase and has been proposed as a wound healing therapy. UV-blue light can activate Opsin 1-SW, Opsin 2, Opsin 3, Opsin 4, and Opsin 5 receptors, triggering biological responses, but their role in human skin physiology is unclear.

Materials and Methods: Localization of Opsins was analyzed in situ in human skin derived from face and abdomen by immunohistochemistry. An ex vivo human skin wound healing model was established and expression of Opsins confirmed by immunohistochemistry. The rate of wound closure was quantitated after irradiation with blue and red light and mRNA was extracted from the regenerating epithelial tongue by laser micro-dissection to detect changes in Opsin 3 (OPN3) expression. Retention of the expression of Opsins in primary cultures of human epidermal keratinocytes and dermal fibroblasts was confirmed by qRT-PCR and immunocytochemistry. Modulation of metabolic activity by visible light was studied. Furthermore, migration in a scratch-wound assay, DNA synthesis and differentiation of epidermal keratinocytes was established following irradiation with blue light. A role for OPN3 in keratinocytes was investigated by gene silencing.

Results: Opsin receptors (OPN1-SW, 3 and 5) were similarly localized in the epidermis of human facial and abdominal skin in situ. Corresponding expression was confirmed in the regenerating epithelial tongue of ex vivo wounds after 2 days in culture, and irradiation with blue light stimulated wound closure, with a corresponding increase in $O P N 3$ expression. Expression of Opsins was retained in primary cultures of epidermal keratinocytes and dermal fibroblasts. Both blue and red light stimulated the metabolic activity of cultured keratinocytes. Low levels of blue light reduced DNA synthesis and stimulated differentiation of keratinocytes. While low levels of blue light did not alter keratinocyte migration in a scratch wound assay, higher levels inhibited migration. Gene silencing of OPN3 in keratinocytes was effective (87\% reduction). The rate of DNA synthesis in OPN3 knockdown keratinocytes did not change following irradiation with blue light, however, the level of differentiation was decreased.

Conclusions: Opsins are expressed in the epidermis and dermis of human skin and in the newly regenerating epidermis following wounding. An increase in OPN3 expression in the epithelial tongue may be a potential mechanism for the stimulation of wound closure by blue light. Since keratinocytes and fibroblasts retain their expression of Opsins in culture, they provide a good model to investigate the mechanism of blue light in wound healing responses. Knockdown of OPN3 led to a reduction in early differentiation of keratinocytes following irradiation with blue light, suggesting OPN3 is required for restoration of the barrier function. Understanding the function and relationship of different photoreceptors and their response to specific light parameters will lead to the development of reliable light-based therapies for

\footnotetext{
Current institution of Charles Mignon: Department of Biomedical Engineering, University Medical Center Groningen, Groningen, The Netherlands.

Current institution of Bianca Raafs: Interchemie werken 'De Adelaar' B.V., LA Waalre, The Netherlands.

Conflict of Interest Disclosures: All authors have completed and submitted the ICMJE Form for Disclosure of Potential Conflicts of Interest and have disclosed the following: Dr. Castellano-Pellicena American Society for Laser Surgery \& Medicine; award Best of photobiomodulation session during the ASLMS conference 2017. This study was conducted under the European Marie-Curie Actions Programme, Grant agreement no. 607886, Irene Castellano Pellicena and Charles Mignon were Early Stage Researchers and Natallia E. Uzunbajakava, Vladimir A. Botchkarev and M. Julie Thornton were the members of a scientific supervisory team. http://www.skinlight-classic.eu/ about classic/.

Contract grant sponsor: European Commission 7th Framework Programme for Research and Technical Development Marie Curie Innovative Training Networks (ITN); Contract grant number: 607886 .

${ }^{*}$ Correspondence to: M. Julie Thornton, Centre for Skin Sciences, University of Bradford, Bradford, United Kingdom. Email: m.j.thornton@bradford.ac.uk

Accepted 6 August 2018

Published online in Wiley Online Library

(wileyonlinelibrary.com).

DOI 10.1002/lsm.23015
} 
cutaneous wound healing. Lasers Surg. Med. C 2018 Wiley Periodicals, Inc.

Key words: photobiomodulation; blue light; red light; Opsins; OPN1-SW; OPN3; OPN5; epidermal keratinocytes; ex vivo wound healing and epidermal barrier

\section{INTRODUCTION}

Photobiomodulation (PBM) as a clinical application for wound healing is impaired by a lack of complete understanding of all involved molecular targets [1], which include cytochrome c oxidase (CCO) [2], nitrozated proteins $[3,4]$, flavoproteins generating reactive oxygen species (ROS) [5-7] and light-activated calcium channels [8]. Previously it was reported that interaction of CCO with red light [2] induces a shift in the redox potential of the cell, inducing transcriptional changes modulating proliferation, metabolism and motility, all important in wound healing [9].

Currently, there is growing interest in the Opsins (OPNs) which are G-protein coupled receptors (GPCRs), as potential targets for light therapy. In humans, the visual OPNs are OPN1 and OPN2 (rhodopsin) which are the main photoreceptors in the eye [10]. Human OPN1 is further subdivided into three forms, OPN1-SW (short wavelength), OPN1-MW (medium wavelength), and OPN1-LW (long wavelength) which are found in cones while OPN2 is found in rods. The maximum absorption spectrum for OPN1-SW is in the violet-blue range (420 $440 \mathrm{~nm}$ ), while OPN2 is activated by cyan light $(496 \mathrm{~nm})$ in the eye [11]. Furthermore, there is increasing evidence that OPN2 in the skin is activated by UVA-violet light in humans [12,13] and mice [14]. There are also non-visual OPNs, which are OPN3 (encephalopsin), OPN4 (melanopsin), and OPN5 (neuropsin) with absorption bands that extend over the UV-A-visible spectrum with corresponding peaks at $460 \mathrm{~nm}$ [15], $496 \mathrm{~nm}$ [16], and $380 \mathrm{~nm}$ [17].

In general, red light $(600-760 \mathrm{~nm})$ was reported to stimulate proliferation and migration of human epidermal stem cells [18] and keratinocytes [19], increase human fibroblast numbers from normal skin and hypertrophic scars [20], and encourage migration of fibroblasts from patients with diabetes [21]. This suggests beneficial roles for red light therapy in re-epithelialization and extracellular matrix formation during wound healing.

In contrast, blue light $(450-490 \mathrm{~nm})$ was reported to inhibit proliferation of neonatal foreskin keratinocytes at $33 \mathrm{~J} / \mathrm{cm}^{2}$ with onset of apoptosis at higher doses $(100 \mathrm{~J} /$ $\mathrm{cm}^{2}$ ) [3]. Blue light was also reported to induce early differentiation of keratinocytes at low doses $\left(5 \mathrm{~J} / \mathrm{cm}^{2}\right)$, while higher settings $\left(66\right.$ and $\left.100 \mathrm{~J} / \mathrm{cm}^{2}\right)$ induced late differentiation [3]. Reduced proliferation and metabolic activity has also been observed in primary cultures of human dermal fibroblasts after irradiation with blue light [6,22]. Recently, Mignon et al. demonstrated a stimulatory effect with a single treatment of $450 \mathrm{~nm}$ light at $2 \mathrm{~J} / \mathrm{cm}^{2}$ on metabolic activity of dermal fibroblasts, with suppressive action following additional irradiations [23].
In a scratch-wound assay, high-dose blue light $\left(55 \mathrm{~J} / \mathrm{cm}^{2}\right)$ inhibited fibroblast migration, while lower doses $(2,5$, and $10 \mathrm{~J} / \mathrm{cm}^{2}$ ) had no effect [24].

In wound healing, red light is thought to stimulate proliferation and re-epithelialization, while blue light has an antimicrobial effect to combat infection $[25,26]$. The anti-proliferative effect is the basis for the use of blue light therapy for psoriasis treatment [27-29], while its modulation of dermal fibroblasts has potential for the treatment of hypertrophic scarring in the latter stages of wound healing [30]. Finally, blue light is also able to induce vasodilatation in mice via activation of OPN4, a very important function to ensure the blood flow reaches the wound site, especially during chronic wound healing [31]. Yet, the role of blue light in other stages of wound healing has not been investigated. Here, we suggest a role for lowdose blue light in the early differentiation of keratinocytes to restore the barrier function of the epidermis.

After the discovery of OPN2 expression in murine melanocytes in culture [32], Tutsumi et al. were the first to identify the presence of visual OPNs (OPN1-SW and M/ LW and OPN2) in human facial skin. In humans, there is a growing evidence for photosensitivity of OPNs outside of the image-forming visual system [12,13,15,17,33-35]. Since most Opsins have their absorption peak in the short wavelength region of the optical spectrum, blue, violet, and UV-A light will be the most effective at activating OPNmediated signaling pathways. In human melanocytes, OPN2 was reported to mediate photon reception and signal transduction following UV-A exposure, leading to calcium mobilization and melanogenesis [13]. More specifically, OPN2 mRNA was up-regulated by violet light $(410 \mathrm{~nm})$ in cultured neonatal foreskin keratinocytes [12]. The nonvisual OPN3, a photoreceptor generally found in animals such as the pufferfish [15], has recently been identified in human hair follicles, including the stem cells [33], and it was activated by low-dose blue light to increased proliferation of cultured outer root sheath keratinocytes [33]. Furthermore, OPN4 was responsible for the vasodilatation response to blue light in mice [31]. Collectively, these findings highlight a function for OPNs in human skin, opening new fields of opportunities for developing lightbased therapies specifically targeting OPNs.

The role of OPNs in wound healing has never been addressed. Therefore, the first aim of this study was to investigate the expression and localization of OPNs in human skin from different anatomical regions, and in the regenerating epithelial tongue of an ex vivo human skin wound-healing model. Then, to establish whether blue or red light could stimulate wound closure in an ex vivo woundhealing model, and whether this could be related to expression of OPN3 in the regenerating epidermis. The second aim was to investigate the retention of OPNs in primary epidermal keratinocytes and dermal fibroblasts in culture, and then, to study whether blue or red light can alter functional responses of epidermal keratinocytes important in wound healing, that is, metabolic activity, proliferation, differentiation and migration and if knockdown of the OPN3 gene would affect light-triggered cell behavior. 


\section{MATERIALS AND METHODS}

\section{Expression of Opsins in Human Skin}

Female human skin was obtained with full written consent adhering to the Declaration of Helsinki principles and under human tissue act guidelines. Facelift or abdominoplasty procedures were always performed in the morning and skin processed in the afternoon of the same day. Seven micrometer cryosections ( $n=4$ donors, 44-63 years) were fixed in acetone before blocking with $5 \%$ bovine serum albumin (BSA) and 5\% donkey serum (DKS) in phosphate-buffered saline (PBS) for 1 hour. Primary antibodies were diluted in 1\% BSA and 1\% DKS and incubated overnight at $4{ }^{\circ} \mathrm{C} ; 1: 200$ OPN1-SW (AB5407, Millipore, Amsterdam-Zuidoost, the Netherlands), 1:100 OPN3 (ab66742 for immunohistochemistry (IHC) and ab75285 for immunocytochemistry (ICC), Abcam, Cambridge, UK), 1:200 OPN5 for IHC and 1:500 for ICC (ab199668, Abcam). A negative control (omission of primary antibody) was included in the experimental procedure. Double staining was performed with 1:200 KRT14 (ab51054 or ab7800, Abcam). Incubation with 1:200 Alexa-488 (ab150073, Abcam) and Alexa-647 (ab150115, Abcam) was for 1 hour at $37^{\circ} \mathrm{C}$. Slides were mounted using VECTASHIELD ${ }^{\mathbb{R}}$ containing DAPI (VECTOR). Images were taken using a confocal microscope (Leica Microsystems B.V., Amsterdam, the Netherlands).

\section{Light-Emitting Devices}

The effect of light was investigated using proprietary LED-based devices, where the irradiance, beam homogeneity, temperature of culture plates, and prevention of light leakage between the plates was carefully controlled or monitored [3,23,33,36]. The temperature of the wells was measured with a FLIR A655sc Infrared Camera, $\left(\right.$ FLIR $^{\mathbb{R}}$ Systems, Inc, Meer, Belgium) at the start of the treatment, which was approximately $37^{\circ} \mathrm{C}$, and at the end, when the temperature had gone down to $24^{\circ} \mathrm{C}$.

The impact of selected discreet wavelengths across visible to near-infrared spectrum on metabolic activity of keratinocytes in vitro was investigated using an LEDbased device emitting 447, 505, 530, 655, and $850 \mathrm{~nm}$ wavelengths and housing 24 well-plates [23]. Response of an ex vivo wounded skin model to light was studied using LED-based devices emitting a single wavelength (either 453 or $656 \mathrm{~nm}$ ) [3,33,36]. The $453 \mathrm{~nm}$ LED-based device was also used to study migration, DNA synthesis and differentiation of keratinocytes. Media was refreshed after every light treatment.

Effect of light on an ex vivo human skin wound healing model. An ex vivo wound healing assay was established [37]. Two parallel incisions were made using an in-house double blade scalpel ( $1 \mathrm{~mm}$ wide). Rectangular wounds $\left(60-80 \mathrm{~mm}^{2}\right)$ surrounded by epidermis at both sides were cultured at the air liquid interface (Netwell inserts, Corning,) in phenol red-free DMEM with $10 \%$ foetal bovine serum [38], $250 \mathrm{U} / \mathrm{ml}$ penicillin and $250 \mu \mathrm{g} / \mathrm{ml}$ streptomycin (Pen/Strep), $7.5 \mu \mathrm{g} / \mathrm{ml}$ of amphotericin B and $5 \%$ glutaMAX. Ex vivo wounds were irradiated daily with two proprietary LED-devices emitting $453 \mathrm{~nm}$ light $[3,33,36]$ at $2 \mathrm{~J} / \mathrm{cm}^{2}$ or $656 \mathrm{~nm}$ at $30 \mathrm{~J} / \mathrm{cm}^{2}$. Images were taken using a wide-field microscope (Leica) and wound area was measured using Image $J$ software. Samples were collected at day 0 (immediately after wounding) and day 2 (4hours after light treatment) for IHC of OPNs (see above) and KRT17 (1:200, ab109725, Abcam).

Laser micro-dissection of regenerating epidermis. Cryosections from ex vivo wounds were collected onto membrane slides (Molecular Machines and Industries [MMI]) and stained with the H\&E RNase free kit (MMI). The epithelial tongue was collected into a $0.5 \mathrm{ml}$ diffuser cap collection tube (MMI) with a Nikon T2000 laser capture microscope. RNA isolation was performed using RNeasy Micro Kit (QIAGEN) and RNA amplification with the Arcturus ${ }^{\text {TM }}$ RiboAmp ${ }^{\mathbb{R}}$ PLUS Kit (ThermoFisher Scientific, Carlsbad, CA). RNA quality was confirmed with Agilent 2100 bioanalyzer (Agilent, Santa Clara, CA).

Quantitative real-time PCR (qRT-PCR) [39] was performed using a high-capacity cDNA reverse transcription kit (Applied Biosystems, UK) and SYBR Green Supermix (Applied Biosystems); OPN primers were used at $1 \mu \mathrm{M}$ (Table 1). Level of expression ( $\mathrm{Ct}\left(\mathrm{C}_{\mathrm{t}}{ }^{\Delta \Delta}\right)$ equitation method) was analyzed with respect to the GAPDH housekeeping gene. Human universal reference total RNA (Clontech) was the positive control.

Cell culture of human primary epidermal keratinocytes and dermal fibroblasts. Primary cultures of human epidermal keratinocytes and dermal fibroblasts from healthy females were established [40]. Keratinocytes (passage 2-5) were cultured in serum free supplemented keratinocyte growth medium 2 (Promocell, UK) and Pen/ Strep. Media was changed to EGF-free and phenol red-free starvation media 24 hours before each experiment. Papillary fibroblasts (passage 3-8) were cultured in DMEM

TABLE 1. Primer Sequences of OPN1-SW, OPN3, OPN5, and KRT10 for qRT-PCR

\begin{tabular}{lcrll}
\hline $\begin{array}{l}\text { Accession } \\
\text { number }\end{array}$ & Gene & Primer sequence (sense/antisense) & T $_{\text {ann }}$ \\
\hline NM_001708 & OPN1-SW & 5'CGCCAGCTGTAACGGATACT3'/5'CCGAAGGGCTTACAGATGAC3' \\
NM_014322 & OPN3 & 5'CAATCCAGTGATTTATGTCTTCATGATCAGAAAG3'/ & 63.1 ${ }^{\circ} \mathrm{C}$ \\
& & 5'GCATTTCACTTCCAGCTGCTGGTAGGT3' & \\
NM_181744 & OPN5 & 5'CTAGACGAAAGAAGAAGAAGCTGAGACC3'/5'GCGGTGACAAAAGCAAGAGA3' & 63.1 ${ }^{\circ} \mathrm{C}$ \\
NM_000421.3 & KRT10 & 5'AGCATGGCAACTCACATCAG3'/5'TGTCGATCTGAAGCAGGATG3' & 62.4 ${ }^{\circ} \mathrm{C}$ \\
\hline
\end{tabular}

$\mathrm{T}_{\mathrm{ann}}$, annealing temperature. 
supplemented with $10 \%$ FBS, Pen/Strep, $7.5 \mu \mathrm{g} / \mathrm{ml}$ of amphotericin B and 5\% glutaMAX. Media was changed to $2 \% \mathrm{FBS}$ and phenol red-free starvation media 24 hours before each experiment.

Total RNA from keratinocytes (facial skin, $n=4$ ) and fibroblasts (breast, face, abdomen and scalp skin, $n=8$ ) was isolated and qRT-PCR of OPN1-SW, OPN2, OPN3, and OPN5 performed as previously described. RT-PCR of OPN4 from fibroblast RNA only was performed as previously described [41]. For ICC, cells were cultured in Millicell EZ 8-well glass slides (Merck, UK).

Effect of light on the metabolic activity of keratinocytes. Keratinocytes in 24 well-plates with a black frame (Porvair Science, Wrexham, UK) were irradiated with blue $\left(447 \mathrm{~nm}, 50 \mathrm{~mW} / \mathrm{cm}^{2}, 2 \mathrm{~J} / \mathrm{cm}^{2}\right)$, cyan $(505 \mathrm{~nm}$, $\left.30 \mathrm{~mW} / \mathrm{cm}^{2}, 2 \mathrm{~J} / \mathrm{cm}^{2}\right)$, green $\left(530 \mathrm{~nm}, 30 \mathrm{~mW} / \mathrm{cm}^{2}, 2 \mathrm{~J} / \mathrm{cm}^{2}\right)$, red $\left(655 \mathrm{~nm}, 40 \mathrm{~mW} / \mathrm{cm}^{2}, 30 \mathrm{~J} / \mathrm{cm}^{2}\right)$ or near infrared (NIR) $\left(850 \mathrm{~nm}, 40 \mathrm{~mW} / \mathrm{cm}^{2}, 60 \mathrm{~J} / \mathrm{cm}^{2}\right)$ light using the proprietary LED-based devices [23]. Alamar Blue ${ }^{\circledR}$ (Thermo Fisher Scientific) assay was performed after 24 hours [23] and relative metabolic activity analyzed with respect to corresponding non-irradiated cultures.

Effect of blue light on the migration of keratinocytes. Keratinocytes in 12-well plates were scratched and the proprietary LED-based device, $\left(453 \mathrm{~nm}, 50 \mathrm{~mW} / \mathrm{cm}^{2}\right.$, $2 \mathrm{~J} / \mathrm{cm}^{2}$ ) was used to irradiate keratinocytes. Images at 0 , 12 , and 21 hours after scratching were analyzed using Image $\mathrm{J}$.

Effect of OPN3 silencing and blue light on DNA synthesis and differentiation of keratinocytes. Keratinocytes cultured on $0.5 \%$ gelatin coated coverslips were synchronized [42] before transfection with complexes of lipofectamine RNAiMax (Invitrogen, Bleiswijk, the Netherlands) and smartpool siRNA (50 nM) directed against OPN3 (siOPN3) or non-targeting siRNA control (NTRC) (ON-Targetplus, Dharmacon). After 24 hours, cells were irradiated with $453 \mathrm{~nm}\left(50 \mathrm{~mW} / \mathrm{cm}^{2}, 2 \mathrm{~J} / \mathrm{cm}^{2}\right)$ light and the thymidine analogue EdU $(10 \mu \mathrm{M})$, $\left(\right.$ Click-iT ${ }^{\circledR}$ EdU Imaging Kits, Invitrogen) was added. After 10 hours cells were fixed with $4 \%$ paraformaldehyde (PFA) for double staining of EdU fluorescence (green) and KRT10 expression (red) by ICC (ab76318, Abcam). Images were taken using the slide scanner, Pannoramic MIDI II (3DHISTECH) and Image $J$ was used to quantify the relative percentage of EdU and KRT10 positive cells. Transfection efficiency and KRT10 expression was performed by qRT-PCR (Table 1).

\section{RESULTS}

Opsins are Similarly Expressed in Human Facial and Abdominal Skin

OPN1-SW, OPN3, and OPN5, were all expressed in female facial and abdominal skin (Fig. 1), while OPN2 has previously been shown to be expressed in facial skin in situ [43]. OPN1-SW and OPN3 were expressed throughout the epidermis, with OPN1-SW mainly in the stratum granulosum, while OPN3 was more prominent in the basal layer (where the basal keratinocytes were identified using
KRT14). OPN1SW and OPN3 were also expressed in the dermis. In contrast, the expression of OPN5 was confined exclusively to the basal keratinocytes.

\section{An Ex Vivo Human Skin Wound Healing Model Regenerates New Epithelium With an Induced Expression of KRT17}

An ex vivo human skin wound healing model was established. After two days in culture, the wound edges visibly started to close (Fig. 2) and histology confirmed the formation of a new epithelial tongue from the wound edges. This was further confirmed by the high expression of KRT17 at the wound margins and in the migrating epithelial tongue after 2 days in culture, in comparison to skin biopsies taken immediately after wounding (Fig. $2 \mathrm{~F}$ and $\mathrm{E}$ ).

\section{Opsins are Expressed in the Epithelial Tongue of Human Skin During Wound Repair Ex Vivo, and Light Accelerates Wound Closure}

OPN expression was also demonstrated in the regenerating epithelium of human ex vivo wounds after 2 days in culture. KRT14 was expressed in the new epithelial tongue (Fig. 3). OPN1SW was co-localized with KRT14 in the apical epithelial tongue, while both OPN3 and OPN5 colocalized with KRT14 in the basal epithelial tongue of the regenerating epidermis.

To establish whether light modulates expression of OPNs during wound healing, the epithelial tongue was isolated by laser micro-dissection from non-irradiated biopsies after blue $\left(2 \mathrm{~J} / \mathrm{cm}^{2}\right)$ or $\operatorname{red}\left(30 \mathrm{~J} / \mathrm{cm}^{2}\right)$ light exposure. Low-level blue light $\left(453 \mathrm{~nm} ; 2 \mathrm{~J} / \mathrm{cm}^{2}\right)$ induced $O P N 3$ expression, while red light had no effect.

The speed of closure for each wound was analyzed at day 2 following light treatment. While it was variable between donors, intra-donor response was consistent. Low dose of blue light $\left(2 \mathrm{~J} / \mathrm{cm}^{2}\right)$ increased the rate of closure in Donor 2 and 3, while red light $\left(30 \mathrm{~J} / \mathrm{cm}^{2}\right)$ increased wound closure only in Donor 2 (Fig. 3).

\section{Primary Epidermal Keratinocytes and Dermal Fibroblasts Derived From Human Skin Retain Expression of Opsins in Culture}

Both mRNA and protein expression of OPNs was confirmed in primary human keratinocytes and papillary dermal fibroblasts. Keratinocytes and fibroblasts expressed mRNA and protein for OPN1SW, which was confined to the peri-nuclear region in both cell types (Fig. 4). Likewise, OPN3 mRNA and protein was also expressed in keratinocytes and fibroblasts, although protein expression was diffusely localized in the cytoplasm and cell membrane. Comparison of the relative mRNA expression of OPN3 showed significantly higher levels in keratinocytes compared to fibroblasts. In contrast, OPN5 (both mRNA and protein) was only expressed in keratinocytes. Interestingly, expression of OPN2 was not retained in keratinocytes in culture while it was found in dermal fibroblasts at both mRNA and protein level (data not shown). OPN4 mRNA was not detected in dermal 

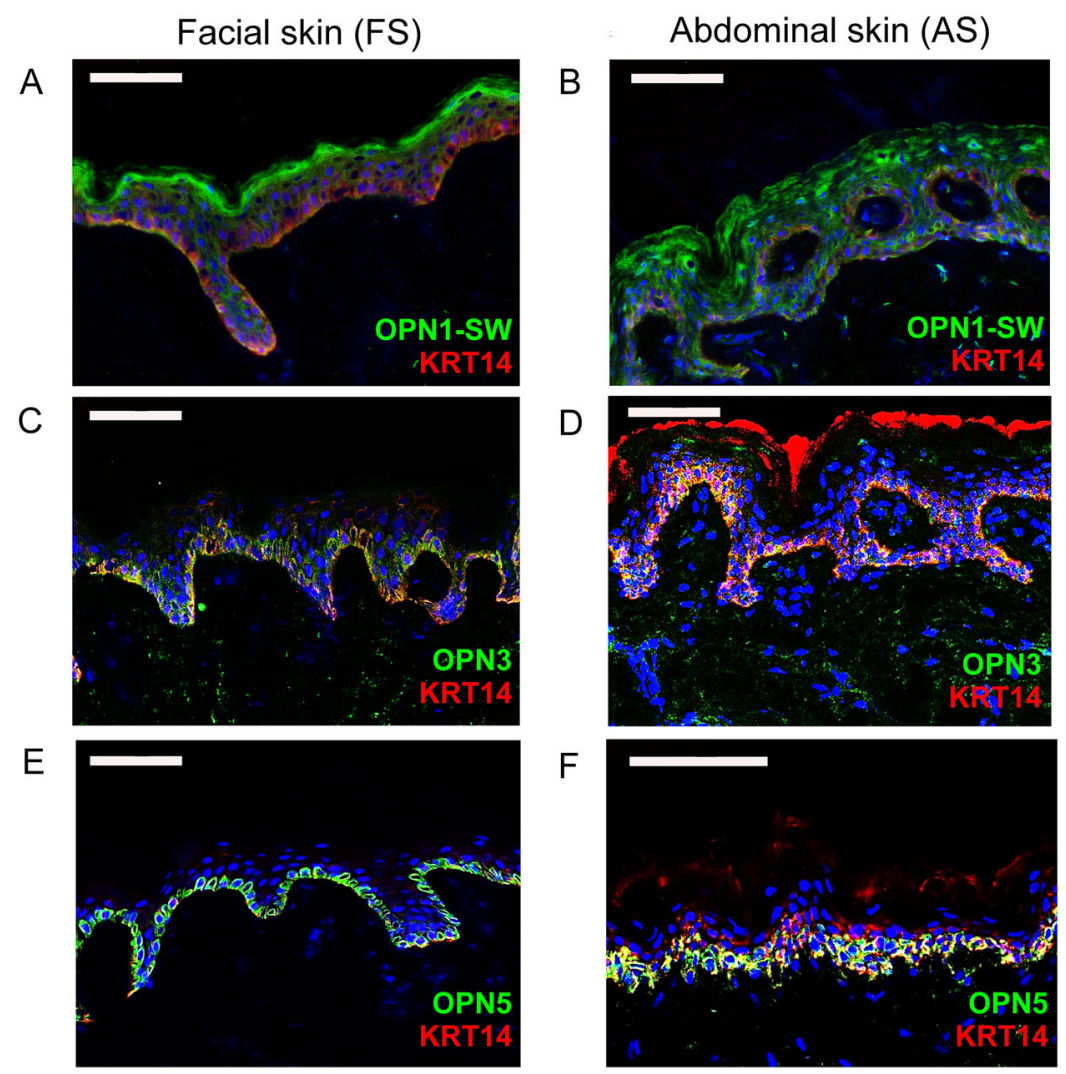

Fig. 1. Localization of OPN1-SW, OPN3 and OPN5 in facial and abdominal human skin by immunohistochemistry. (A) Localization of OPN1-SW (green) and KRT14 (red) in facial skin (FS). (B) Localization of OPN1-SW (green) and KRT14 (red) in abdominal skin (AS). (C) Localization of OPN3 (green) and KRT14 (red) in FS. (D) Localization of OPN3 (green) and KRT14 (red) in AS. (E) Localization of OPN5 (green) and KRT14 (red) in FS. (F) Localization of OPN3 (green) and KRT14 (red) in AS. Yellow denotes co-localization. Nuclei are counterstained with DAPI (blue). Scale bars $=75 \mu \mathrm{M}$. Representative images from four individual donors. Negative control (omission of primary antibody) was included to confirm there was no non-specific binding (data not shown).

fibroblasts in culture so further analysis was not carried out (data not shown).

\section{Light Has a Physiological Effect on Epidermal Keratinocyte Function in Culture}

To establish a physiological effect of light on human keratinocytes, cultures were irradiated with 2 and $30 \mathrm{~J} /$ $\mathrm{cm}^{2}$ of blue, cyan and green light $(447,505$, and $530 \mathrm{~nm})$, as well as $30 \mathrm{~J} / \mathrm{cm}^{2}$ of red light $(655 \mathrm{~nm})$ or $60 \mathrm{~J} / \mathrm{cm}^{2}$ of NIR light $(850 \mathrm{~nm})$ and metabolic activity was quantitated. A statistically significant increase in metabolic activity was stimulated by $2 \mathrm{~J} / \mathrm{cm}^{2}$ of blue, cyan and red light (Fig. 5). However, $2 \mathrm{~J} / \mathrm{cm}^{2}$ of green light, $30 \mathrm{~J} / \mathrm{cm}^{2}$ of blue, cyan and green light and $60 \mathrm{~J} / \mathrm{cm}^{2}$ of NIR had no effect.

Since OPN1-SW and OPN3 have an absorption peak in the blue light wavelength, to further understand their potential role on epidermal keratinocytes, the effect of $2 \mathrm{~J} /$ $\mathrm{cm}^{2}$ of $453 \mathrm{~nm}$ (blue) light on cell morphology, cell differentiation, DNA synthesis and cell migration in a scratch-wound assay was investigated. Cultures of human primary epidermal keratinocytes, following irradiation with $2 \mathrm{~J} / \mathrm{cm}^{2}$ of blue light, showed no changes in morphology and colony formation ability after treatment (Fig. 5). DNA synthesis of keratinocytes was reduced following exposure to low levels of blue light, while differentiation, as shown by expression of KRT10, a marker of early differentiation, was induced (Fig. 5). In contrast, blue light had no effect on keratinocyte migration in a scratchwound assay. To further investigate the effect of blue light on their migratory ability, keratinocytes were exposed to a higher dose $\left(30 \mathrm{~J} / \mathrm{cm}^{2}\right)$ in a scratch-wound assay, which reduced migration as early as 12 hours.

\section{Blue Light-Induced Differentiation of Cultured Epidermal Keratinocytes Is Regulated by OPN3}

Since low-irradiance blue light decreased DNA synthesis and increased differentiation (Fig. 5), the effect of blue light following knockdown of OPN3 was evaluated. The efficiency of OPN3 knockdown was confirmed by an $87 \%$ reduction of OPN3 mRNA in siOPN3 keratinocytes compared to control NTRC (Fig. 6). The reduction in OPN3 protein expression was confirmed by Western Blot 

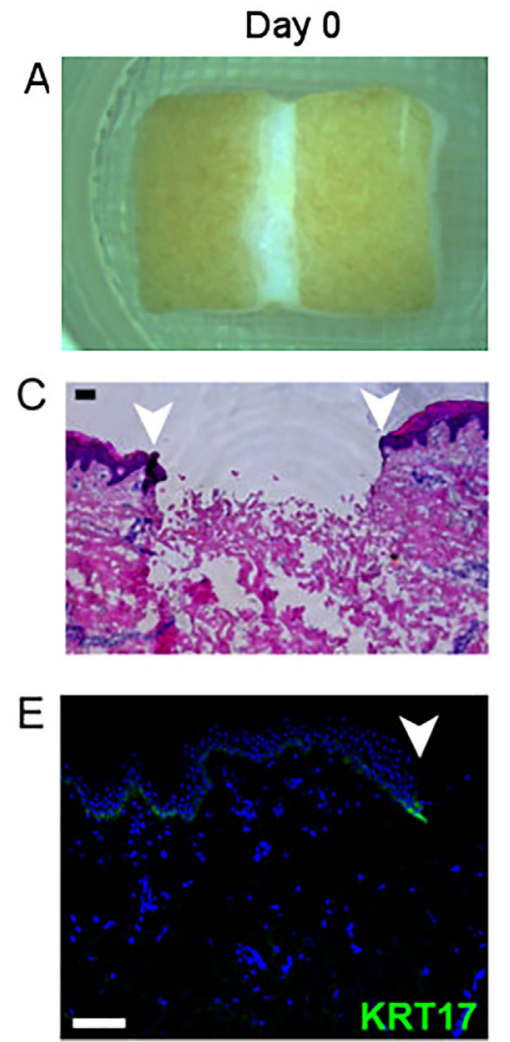

Day 2
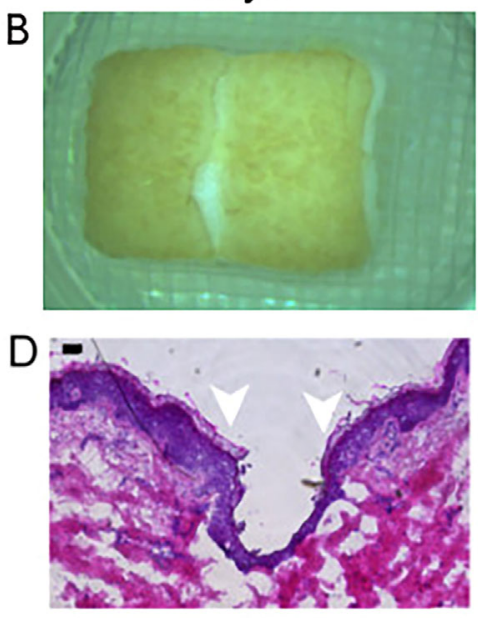

$\mathrm{F}$

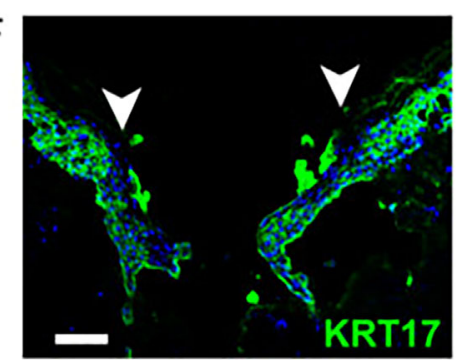

Fig. 2. Human ex vivo wound healing model. (A) Representative image of ex vivo human skin immediately after wounding and (B) two days after wounding and cultured in the air liquid interphase ( $n=3$ donors). (C) Hematoxylin and eosin staining ex vivo human skin immediately after wounding showing removal of epidermis and upper dermis. (D) Hematoxylin and eosin staining of ex vivo wounds after two days in culture. Arrows denote wound edge. (E) KRT17 (green) expression in human skin immediately after wounding. (F) KRT17 (green) expression in ex vivo wounds after two days in culture. Nuclei are counterstained with DAPI (blue). Scale bar $=75 \mu \mathrm{M}$.

$48 \mathrm{~h}$ after silencing demonstrating it had decreased by $61.42 \%$ (data not shown). The expression of KRT10 mRNA did not change, confirming OPN3 knockdown had no effect on keratinocyte differentiation.

Following knockdown of OPN3, keratinocytes irradiated with $2 \mathrm{~J} / \mathrm{cm}^{2}$ of blue light significantly reduced EdU incorporation, a marker of DNA synthesis. However, DNA synthesis was similarly reduced in NTRC keratinocytes by blue light. In contrast, while blue light induced differentiation in NTRC keratinocytes, this effect was abrogated in OPN3 knockdown keratinocytes (Fig. 6).

\section{DISCUSSION AND CONCLUSIONS}

Since the first sequence of an Opsin, bovine rhodopsin, was determined by protein sequencing in 1982, more than 1,000 opsins have been identified, 9 of them are present in the human genome [10].

Beyond vision, the OPN family have been implicated in other functions. For example, OPN4 is considered to be a circadian clock photoentrainment molecule in mammals [44-47], while OPNs have also been implicated in functions related to temperature regulation [48-50]. Fascinatingly, following the work of Miyashita et al. describing the expression of OPN2 and cone opsins in murine melanocytes [32], the presence of visual opsins, OPN1 and OPN2 that mediate day-light (color) and twilight vision in the human eye [51] has been reported in human facial epidermis [43], suggesting a potential light-sensitive role in the skin. Indeed, since then, the biological role of OPNs in light photoreception in skin is emerging as a new, exciting field in PBM [12,13,33-35], as well as in other tissues beyond the eye $[15,17,31,52,53]$.

Interestingly, we have observed the presence of OPN1SW, which is activated by violet-blue light (420$440 \mathrm{~nm}$ ) [10], a wavelength that does not penetrate very far into the skin [54], not only in the superficial layers of the epidermis in agreement with Tutsumi et al., but strikingly also in the papillary dermis. In contrast, the expression of OPN3 (with an absorption peak at a longer wavelength of $460 \mathrm{~nm}$ ) was mainly confined to the stratum basale, albeit lower expression was also seen in the suprabasal layer. This is partially in agreement with localization of OPN3 in human scalp [33], where expression was higher in differentiated layers of the epidermis compared to facial or abdominal skin in this study. The expression of OPN3 in basal keratinocytes may be explained by its absorption at a slightly longer wavelength $(465 \mathrm{~nm})$ [15], compared to OPN1-SW [10]. However, we 
A

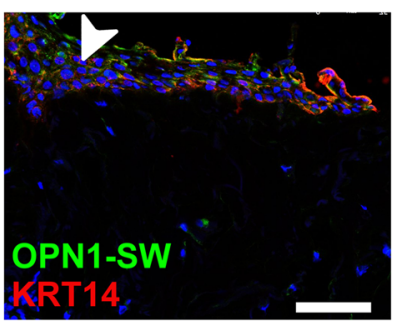

C

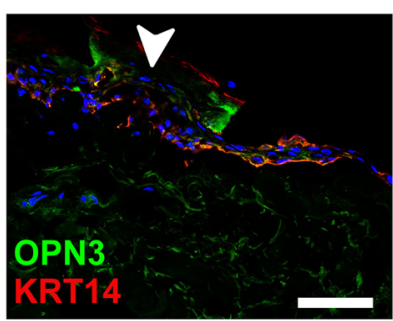

B

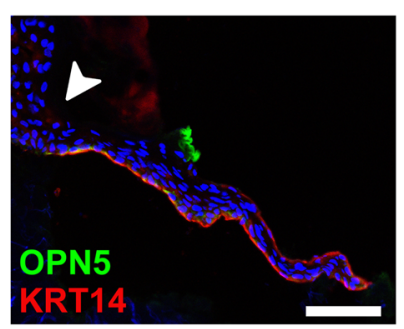

D

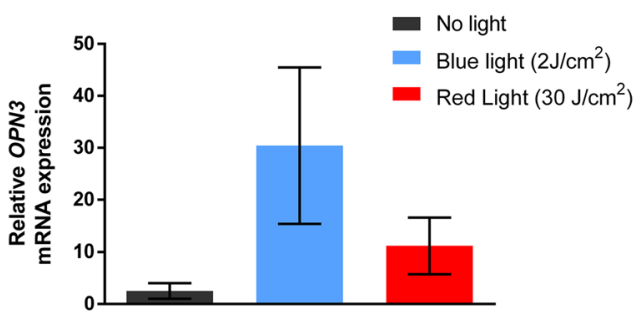

E

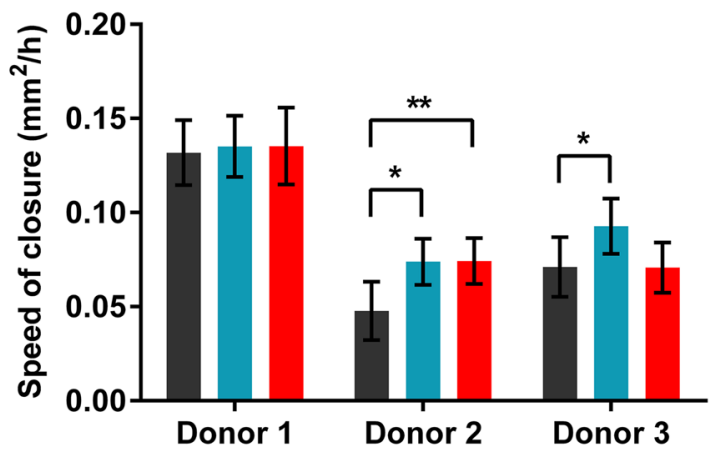

no light

Blue light $\left(2 \mathrm{~J} / \mathrm{cm}^{2}\right)$

Red light $\left(30 \mathrm{~J} / \mathrm{cm}^{2}\right)$

Fig. 3. Expression of OPNs and effect of blue and red light during re-epithelialization. (A-C) Localization of KRT14 (red) and OPN (green); OPN1-SW (A), OPN5 (B) and OPN3 (C) in the newly formed epithelial tongue of ex vivo wounds after two days in culture. Arrows denote growth of new epidermis. Nuclei are counterstaining with DAPI. Scale bar $=75 \mu \mathrm{M}$. Representative images from 4 individual donors. (D) Relative mRNA expression of OPN3 in the newly formed epithelial tongue after irradiation with blue light $\left(2 \mathrm{~J} / \mathrm{cm}^{2}\right)$ or red light $\left(30 \mathrm{~J} / \mathrm{cm}^{2}\right)$. Data was calculated relative to GAPDH and presented as the mean of the normalized relative expression of $n=2-3$ wounds \pm SEM. (E) Mean of speed of closure of human ex vivo wounds after two days in culture \pm SD $(n=5-7$ wounds per donor). Wounds were treated twice ( 24 hours interval) with blue $\left(2 \mathrm{~J} / \mathrm{cm}^{2}\right)$ and red light $\left(30 \mathrm{~J} / \mathrm{cm}^{2}\right) .{ }^{*} P<0.05,{ }^{* *} P<0.01$ in a two-way ANOVA.

are tempted to suggest that one cannot simply state that the expression of Opsins as a function of depth in the skin is solely guided by the wavelength-dependence of their absorption peak, as violet light absorbing OPN1-SW was also found in papillary dermal fibroblasts. As for OPN5, a UV-absorbing receptor [55], we believe that this is the first time its expression in human skin in situ is reported (Fig. 1). Localization of OPN5 was restricted exclusively to the basal layer of the epidermis as illustrated by its perfect co-localization with KRT14, a specific marker of basal keratinocytes. Although its peak is within the UV-A spectrum [55], which again is thought not to not penetrate deep in the skin, OPN5 was expressed in the epidermal layer containing proliferative keratinocytes, stem cells and melanocytes [56-58], where protection against UV light is critical. Since OPN5 is expressed in cultured neonatal foreskin melanocytes [41], our finding could indicate a potential role of OPN5 in the UV response of basal keratinocytes, and cross-talk with melanocytes to regulate skin protection and prevent DNA damage [59].

One could speculate that OPNs might be predominately expressed in light-exposed areas of human skin, for example, the face. This hypothesis is in agreement with the expression level of OPN1-LW and OPN4 in human skin, which is higher in sun-exposed skin. However, it is not the case for OPN5, which was more highly expressed in non sun-exposed skin, while $O P N 1-S W,-M W, O P N 2$ and $O P N 3$ did not show any difference with anatomical regions [14]. In this study, OPN1-SW, OPN3, and OPN5 demonstrated a similar pattern of localization in abdominal skin and sun-exposed facial skin (Fig. 1). The expression of OPN1-SW and OPN3 suggest they may 

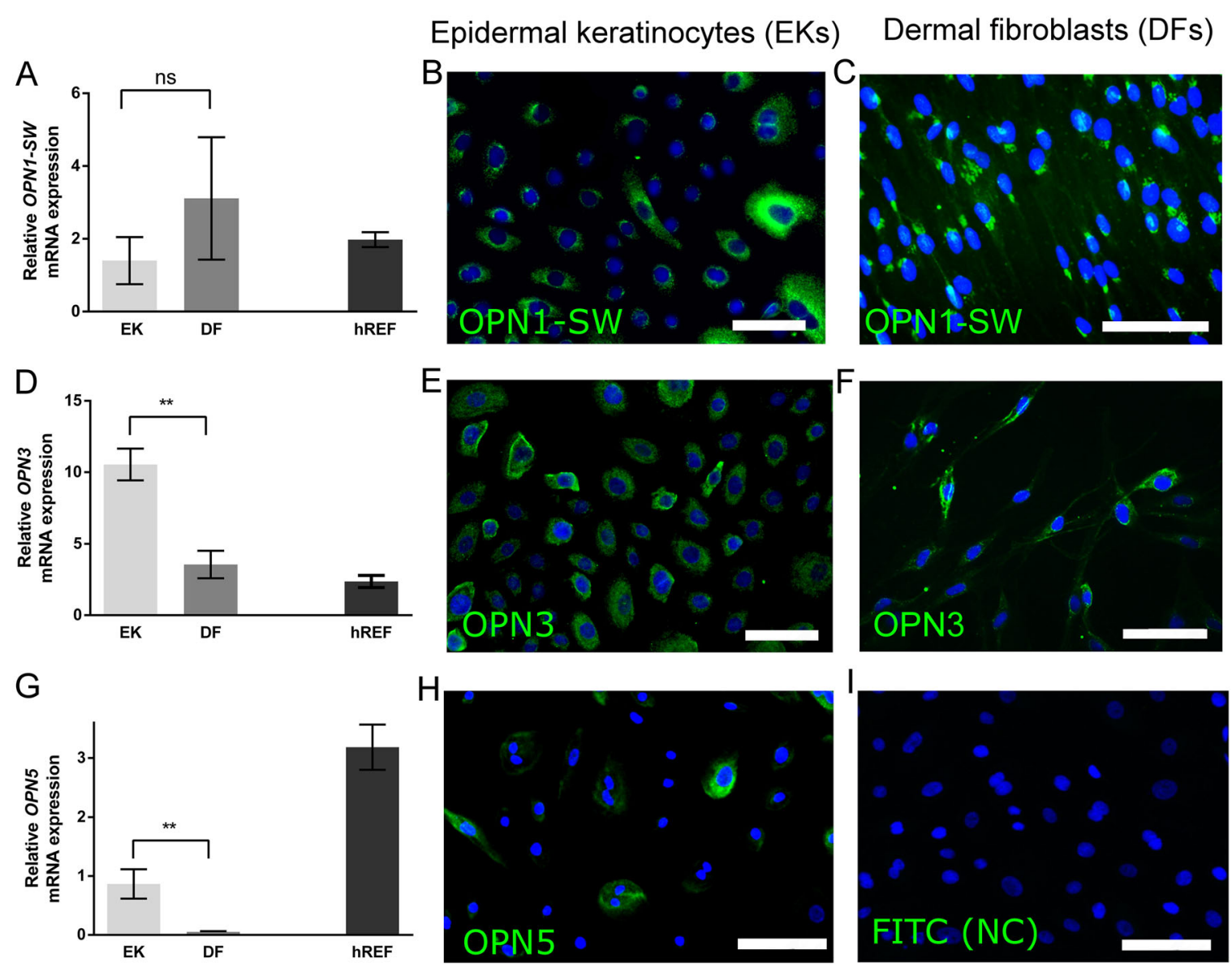

Fig. 4. Expression of Opsin mRNA and protein in cultures of human primary epidermal keratinocytes and dermal fibroblasts. Relative expression of mRNA in EKs $(n=4)$ and DFs $(n=8)$ for (A) OPN1-SW, (D) OPN3, (G) OPN5 \pm SEM quantitated by qRT- PCR; human reference RNA (hREF) was used as a positive control. ${ }^{* *} P$-value $<0.01$ and ns represent not significant using Mann-Whitney test. Representative images of (B) OPN1-SW (green), (E) OPN3 (green), and (H) OPN5 (green) protein localization in EKs by immunofluorescence; nuclei are counterstained with DAPI (blue). Representative images of (C) OPN1-SW (green) and (F) OPN3 (green), protein localization in DFs by immunofluorescence. (I) Negative control (no primary antibody) in DFs. Nuclei are counterstained with DAPI (blue). Scale bar $=75 \mu \mathrm{M}$.

have biological functions beyond light reception in nonphotoreceptive cells, or they are vestigial evolutionary remnants from mechanisms developed in the prehistoric times of "naked apes" and have remained well conserved even after humans had experienced dramatic changes in lifestyle, such as sun protection with clothing. The fact that OPN3 has been found in a range of non-photoreceptive tissues including the brain, testis and liver [10] also supports these theories. Finally, more research into the role of the expression of OPN5 in sun-exposed skin is required. The fact that OPN5 is specifically expressed only in the basal layer of the epidermis means that any sundamage to the cells in this layer could affect the expression of OPN5.

Ex vivo cultures recapitulate the physiology of keratinocytes in vivo as communication with other skin cell, for example, fibroblasts, melanocytes, immune cells. An ex vivo human skin wound healing model was established to investigate OPNs expression in the regenerating epithelial tongue and if red or blue light could modulate wound closure. The expression of KRT17 confirmed the regeneration of a new epithelial tongue in ex vivo wounds (Fig. 2). OPN1-SW, OPN3, and OPN5 were all expressed in this newly formed epidermis, suggesting their role in reepithelialization. Interestingly, OPN expression here closely resembled that of normal epidermis. While OPN1-SW was highly expressed in the apical migrating keratinocytes, OPN3 and OPN5 were highly expressed in basal keratinocytes (Fig. 3). Furthermore, expression of $O P N 3$ in the epithelial tongue was upregulated following exposure to low-level blue light, but not red light (Fig. 3). Since OPN3's peak absorption is in the blue spectra [15], it does not feel utterly non-logical that red light does not stimulate it. A major role of the epidermis is to provide a protective barrier against external pathogens and prevent trans-epidermal water loss (TEWL) [60]. Disruption of the 
A

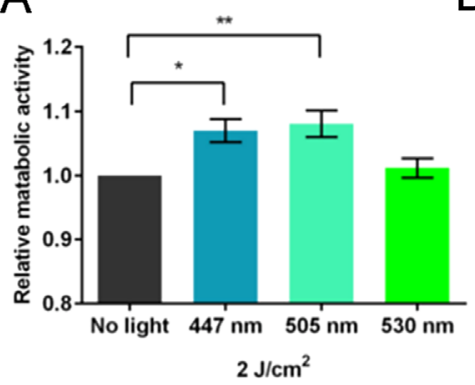

D Control EKs

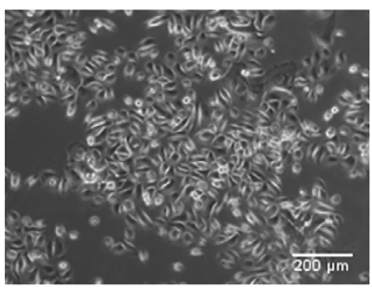

F DNA synthesis

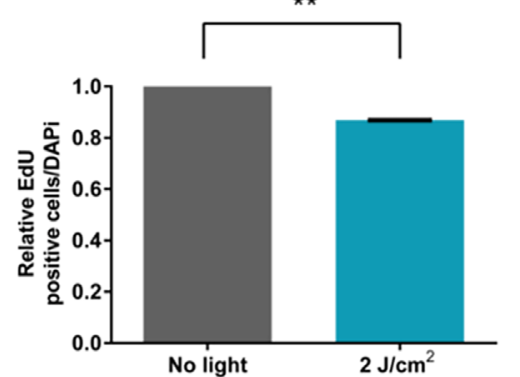

Metabolic activity

B

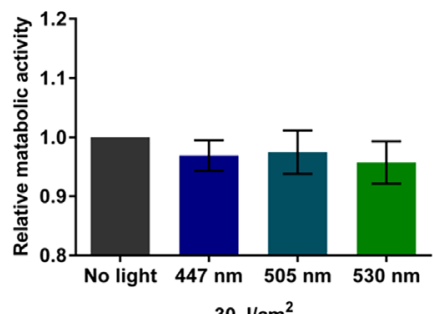

$30 \mathrm{~J} / \mathrm{cm}^{2}$
C

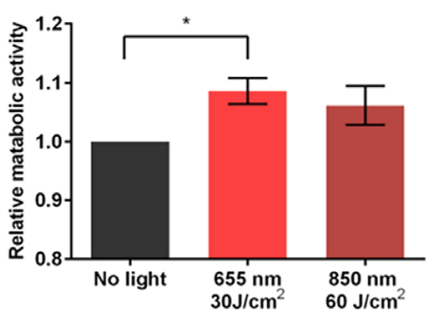

E Blue light (2J/cm2) EKs

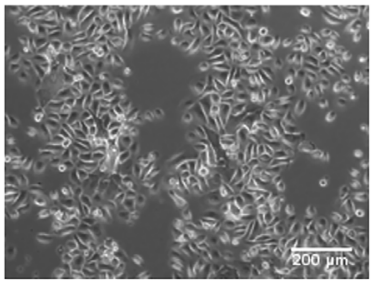

G Early differentiation

$\mathrm{H}$ Migration

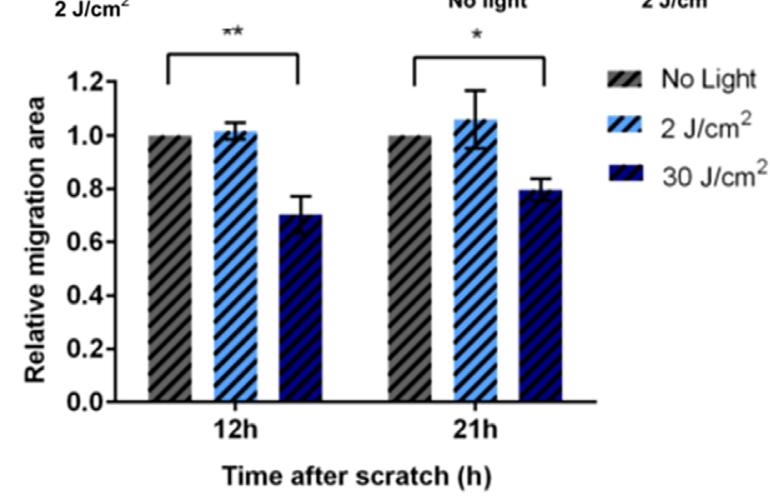

Fig. 5. Effect of visible light of different wavelengths and doses on metabolic activity, proliferation, differentiation and migration of epidermal keratinocytes. (A-C) Relative metabolic activity of EKs following exposure to (A) $2 \mathrm{~J} / \mathrm{cm}^{2}$ of short wavelength light 447 (blue), 505 (cyan) and 530 (green) nm, (B) $30 \mathrm{~J} / \mathrm{cm}^{2}$ of short wavelength light 447 (blue), 505 (cyan) and 530 (green) $\mathrm{nm}$, and (C) long wavelength light $\left(655 \mathrm{~nm}\left(\right.\right.$ red at $\left.30 \mathrm{~J} / \mathrm{cm}^{2}\right)$ and $850 \mathrm{~nm}\left(\mathrm{NIR}\right.$ at $\left.60 \mathrm{~J} / \mathrm{cm}^{2}\right)$. Data presented as mean of $n=4$ donors \pm SEM ${ }^{*} P<0.05$ and ${ }^{* *} P>0.01$ using one way ANOVA. (D-E) Images of control (nontreated) (D) and blue light treated $\left(2 \mathrm{~J} / \mathrm{cm}^{2}\right)(\mathbf{E})$ EKs. Scale bar $=200 \mu \mathrm{m}$. (F) Quantification of DNA synthesis by EdU incorporation of EKs after irradiation with $453 \mathrm{~nm}$ (blue) light at $2 \mathrm{~J} / \mathrm{cm}^{2}$. Data presented as relative mean of EdU positive cells/DAPI \pm SEM. ${ }^{* *} P>0.01$ using unpaired $t$-test. (G) Quantification of differentiation by KRT10 expression after irradiation with $453 \mathrm{~nm}$ (blue) light at $2 \mathrm{~J} / \mathrm{cm}^{2}$. Data presented as relative mean of KRT10 positive cells/DAPI $(n=3$ donors $) \pm$ SEM. ${ }^{*} P>0.05$ using unpaired $t$-test. (H) Quantification of migration 12 and 21 hours after scratchwounding monolayers of EKs. EKs were irradiated with $453 \mathrm{~nm}$ (blue) light at $2 \mathrm{~J} / \mathrm{cm}^{2}$ or $30 \mathrm{~J} / \mathrm{cm}^{2}$ immediately after scratching. Data presented as relative mean of closure of $n=3$ donors \pm SEM. ${ }^{*} P<0.05,{ }^{* *} P<0.01$ using two-way ANOVA. 


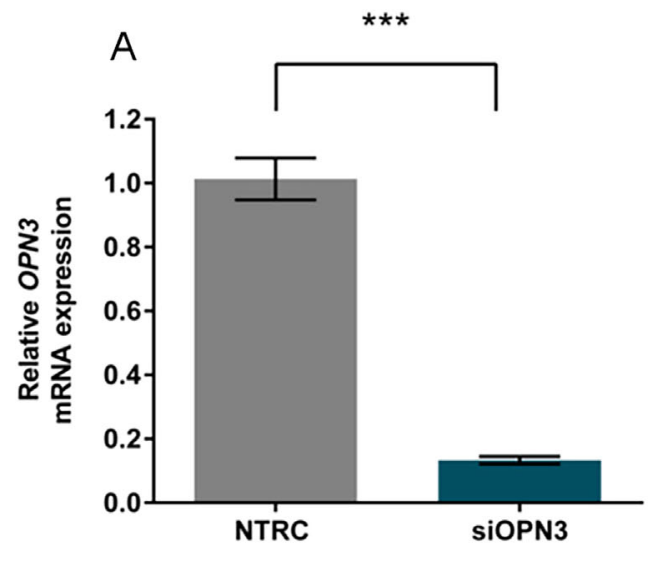

B

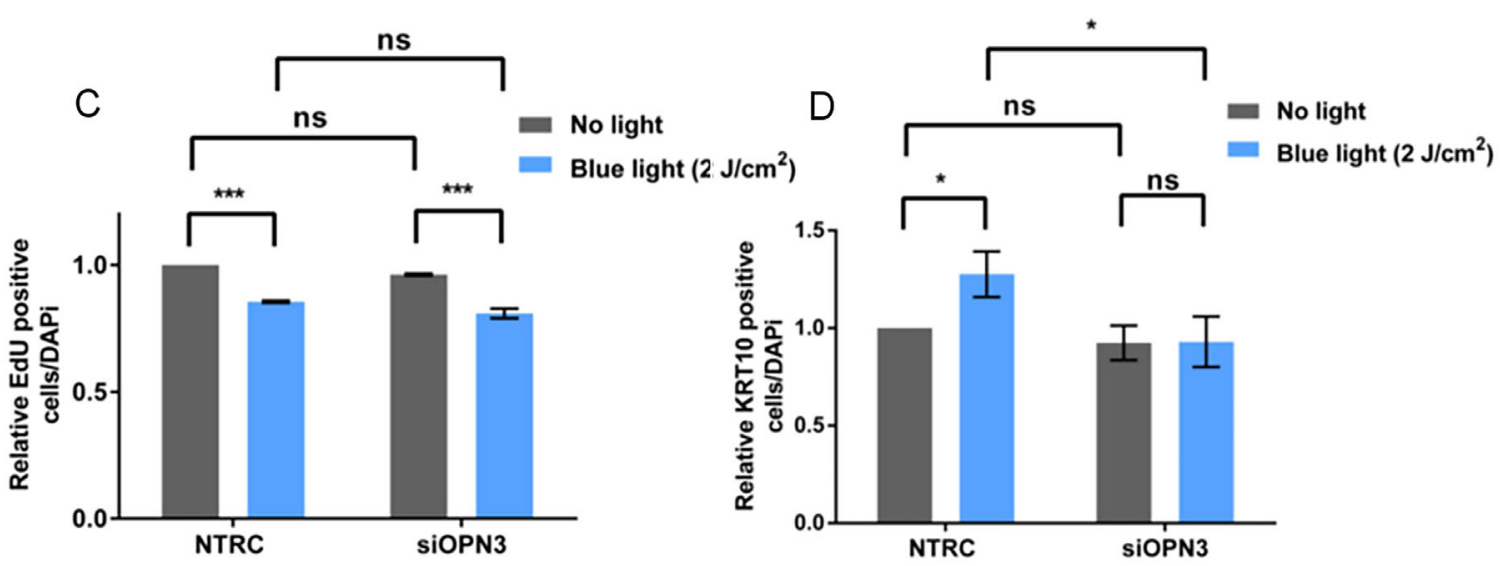

Fig. 6. Effect of blue light on proliferation and differentiation of OPN3 silenced human epidermal keratinocytes. (A-B) Relative mRNA expression of OPN3 (A) and KRT10 (B) in EKs treated with non-target siRNA control (NTRC) or siRNA against OPN3 for 24 hours. Data presented as mean relative expression of $n=3$ donors \pm SEM. ${ }^{* * *} P>0.001$ using a unpaired $t$-test. (C) DNA synthesis of EKs treated with NTRC or siRNA against OPN3 for 24 hours and irradiated with $453 \mathrm{~nm}$ light at $2 \mathrm{~J} /$ $\mathrm{cm}^{2}$. Data presented as the mean relative EdU positive cells/DAPI of EKs \pm SEM. ${ }^{* * *} P<0.001$ and ns indicates not significant using two-way ANOVA. (D) KRT10 expression of epidermal keratinocytes treated with non-target siRNA control or siRNA against OPN3 for 24 hours and irradiated with $453 \mathrm{~nm}$ light at $2 \mathrm{~J} / \mathrm{cm}^{2}$. Data presented as the mean relative KRT10 positive cells/ DAPI of EKs ( $n=3$ donors) \pm SEM. ${ }^{*} P>0.05$ using two-way ANOVA.

barrier leads to a series of cellular responses, including changes in gene expression. In order for keratinocytes to migrate they must undergo an epithelial to mesenchyme transition (EMT), where proteins such as vimentin are upregulated and others, for example, E-cadherin are downregulated [61]. While migration, proliferation and differentiation of keratinocytes is vital in wound repair, so too is restoration of the functional barrier requiring the synthesis of key components such as cross-linked lipids and proteins, for example, filaggrin and loricrin [62]. The epidermis also acts as a first-response immune organ when the barrier is breached and the upregulation of antimicrobial peptides (AMP) such as cathelicidin and defensins have also been shown to accelerate wound repair [63]. At present, a role for OPN3 in any these responses is unknown, but the increase in OPN3 mRNA expression might indicate a functional response of OPN3 to blue light during human wound healing and warrants further investigation.
While investigating the effect of light on wound closure we noted that the differences in speed of closure highlighted donor variability in human skin ex vivo.

While all biopsies were taken from the same anatomical region of women of a similar age, biological variability, including hormonal fluctuations between different donors may explain the variability in wound closure [64]. Donor variability has been previously reported in skin ex vivo cultures, which may be attributed to different inflammatory responses induced by tissue removal [65] or different circadian rhythms as a consequence of the different environmental cues that individual donors have been exposed to (e.g. food intake, shift work, stress, smoking etc.), which can impact on epidermal stem cell function $[42,66]$. On a positive side, the low variation within donors $\left(<0.017 \mathrm{~mm}^{2} / \mathrm{h}, \mathrm{SD}\right)$ supported reproducibility of the developed model. Therefore, we have chosen to study the effect of light independently in each of the donors. The response to light of different donors followed a similar 
trend (Fig. 3); interestingly, low-dose blue or red light induced wound closure in Donor 2 and blue light also induced wound closure in Donor 3. Despite this observation, our thinking is that the molecular mechanisms activated by blue and red light are likely to be highly different, with $\mathrm{CCO}$ as the main photoreceptors of red light [2], while OPNs might be a new molecular target triggered by shorter wavelengths of light, in particular OPN3 which is suggested to be a potential light-therapeutic target as it is upregulated after blue light treatment in the epithelial tongue of the ex vivo wounds (Fig. 3).

In order to further investigate the molecular mechanisms of OPNs and light reception in skin cells, their expression in primary cultures of keratinocytes and fibroblasts was characterized. While mRNA expression of $O P N 1 S W, O P N 3$, and OPN5 in keratinocytes has previously been reported [41], expression in dermal fibroblasts, and expression at the protein level has not yet been established until now. We confirmed that primary keratinocytes express OPN1-SW, OPN3, and OPN5 at both the mRNA and protein level.

Furthermore, here we report for the first time that cultured dermal fibroblasts from different anatomical regions express OPN1-SW, OPN2 and OPN3, but not OPN4 and OPN5 (Fig. 4). Interestingly, expression of OPN3 mRNA was higher in keratinocytes compared to fibroblasts (which might reflect their sensitivity to light coupled to a deeper anatomical position of the latter ones and thus lower "chance" to interact with blue-colored photons), however, there was no difference in expression of OPN1-SW between these two cell types. In cultured keratinocytes and fibroblasts, OPN1-SW was confined to the peri-nuclear region. Intriguingly, the same localization profile is found in human sperm [48]. The authors of this study highlighted a role for OPN4 in sperm thermotaxis mediated by phospholipase C (PLC), due to its specific localization around the postnuclear cap, which is the location of the non-acrosomal $\mathrm{Ca}^{2+}$ store involved in PLC signaling. Therefore, the role of OPN1-SW in human skin may be related to temperature sensing, however, perinuclear OPN4 localization has also been observed in normal and melanoma cell lines [67], where it can respond to UV-A light in mice [14] indicating a possible photoreceptor activity of OPNs located in the perinuclear region of cells. In contrast, OPN3 was localized to the plasma membrane and cytoplasm, and OPN5 was seen in the plasma membrane and cytoplasm, however, only in keratinocytes, suggesting functional roles for non-visual OPN3 and OPN5 in human epidermis. In a first screening of OPN expression, OPN4 mRNA was not detected in primary human dermal fibroblasts in culture and therefore further study of this protein was not carried out. However, further investigation into the presence of OPN4 is still required to have the complete picture of OPN expression in human skin.

Since we identified that cultured primary cells maintained OPN expression at mRNA and protein levels, logically in vitro cell cultures should provide a physiologically relevant model to explore the effect of light on human skin. To determine a role in PBM, we sought to establish the effect of blue light on epidermal keratinocytes, since this is where most OPNs have their absorption spectra. What also supported our choice is that OPN3 and OPN5 were more highly expressed in epidermal keratinocytes than dermal fibroblasts (Fig. 3). For the sake of completeness (as highly used in PBM) red and NIR light was also included in the study on metabolic activity. Interestingly, wavelengths of blue, cyan and the red part of the spectrum (447, 505, and $654 \mathrm{~nm}$ ) stimulated metabolic activity demonstrating the overall ability of keratinocytes to respond to visible light. Since an increase in metabolic activity was stimulated at 447 and $505 \mathrm{~nm}$, but not $530 \mathrm{~nm}$, this may be mediated by OPN1-SW or OPN3, as their peaks of absorption are 430 and $465 \mathrm{~nm}$, respectively. One cannot exclude that OPN5 may also be involved, since its photoproduct absorbs light at $447 \mathrm{~nm}$ following photoconversion after UV stimulation [17].

Low-level blue light decreased DNA synthesis, but stimulated keratinocyte differentiation (Fig. 5), suggesting the increase in metabolic activity does not translate into increased proliferation, but rather into restoration of the epidermal barrier. Indeed, low-dose blue light did not modulate migration of keratinocytes, while a high dose of $30 \mathrm{~J} / \mathrm{cm}^{2}$ had an inhibitory effect, in line with reports on cultured dermal fibroblasts $[24,68]$.

To establish whether effects of blue light on keratinocytes are modulated via OPNs, the consequence of silencing OPN3 was evaluated. OPN3 was not essential for DNA synthesis or differentiation of non-irradiated keratinocytes (Fig. 6). Furthermore, the decrease in DNA synthesis induced by blue light also occurred when OPN3 was silenced, suggesting the decrease in proliferation in response to blue light is not regulated by OPN3. However, blue light increased the expression of KRT10, which was abrogated by silencing OPN3, suggesting this effect is at least partially modulated by OPN3. One possible downstream mechanism triggered by OPN3 could act via cAMP and $\mathrm{Ca}^{2+}$ signaling [69], which can activate the CREB and the Ras signaling pathways. What supports this hypothesis is that knockdown of OPN3 blocked the calcium flux and the phosphorylation of CREB and $\mathrm{Ca}^{2+} /$ calmodulin-dependent protein kinase II (CAMKII) in non-irradiated and blue lightirradiated human melanocytes [35]. Furthermore, OPN2 has been shown to contribute to the induction of UVdependent $\mathrm{Ca}^{2+}$ mobilization in melanocytes [13]. In human epidermal keratinocytes, a non-canonical OPN2 signaling pathway mediated by Gi protein has been reported [12]

In conclusion, OPNs are expressed in human skin and the regenerating epithelial tongue after wounding, with upregulation of OPN3 expression after blue light irradiation. Furthermore, low-level blue and red light stimulated wound closure in human skin ex vivo, indicating beneficial roles of light during wound healing. The effect of low-level blue light on primary keratinocytes in vitro highlights its potential role in regulating metabolic activity and differentiation, which is certainly of importance for epidermal barrier restoration following the re-epithelialization in wound healing. Gene silencing has identified OPN3 as a 
blue-light receptor regulating keratinocyte differentiation and a potential player in restoring skin barrier functionality. Therefore blue light (at least at low dose levels) may provide beneficial effects in treatments of chronic wounds unable to restore functionality of the epidermal barrier, as well as in other skin conditions such as ichthyosis and atopic dermatitis [70].

\section{ACKNOWLEDGMENTS}

This study was supported by the European Commission 7th Framework Programme for Research and Technical Development - Marie Curie Innovative Training Networks (ITN), Grant agreement no.: 607886. The authors also acknowledge the support of the American Society for Laser Surgery \& Medicine in the form of an award Best of photobiomodulation session̈ during the ASLMS conference 2017.

\section{AUTHORS' CONTRIBUTIONS}

Dr. Irene Castellano-Pellicena designed and conducted experiments, analyzed the data and wrote the manuscript. Dr. Charles Mignon created the light-based devices and contributed to the experimental design and data interpretation of the light-based experiments. Bianca Raafs assisted in performing qRT-PCR, cell isolation and culture. Dr. ir. Natallia E. Uzunbajakava and Dr. M. Julie Thornton jointly conceived and supervised the study, assisted in data interpretation and edited the manuscript. Prof. Vladimir A. Botchkarev, MD, contributed to editing the manuscript.

\section{REFERENCES}

1. Mignon C, Botchkareva NV, Uzunbajakava NE, Tobin DJ. Photobiomodulation devices for hair regrowth and wound healing: A therapy full of promise but a literature full of confusion. Exp Dermatol 2016;25(10):745-749.

2. Karu T. Primary and secondary mechanisms of action of visible to near-IR radiation on cells. J Photochem Photobiol B 1999;49(1):1-17.

3. Liebmann J, Born M, Kolb-Bachofen V. Blue-light irradiation regulates proliferation and differentiation in human skin cells. J Invest Dermatol 2010;130(1):259-269.

4. Opländer C, Deck A, Volkmar CM, et al. Mechanism and biological relevance of blue-light $(420-453 \mathrm{~nm})$-induced nonenzymatic nitric oxide generation from photolabile nitric oxide derivates in human skin in vitro and in vivo. Free Radic Biol Med 2013;65:1363-1377.

5. Nakashima Y, Ohta S, Wolf AM. Blue light-induced oxidative stress in live skin. Free Radic Biol Med 2017;108:300-310.

6. Opländer C, Hidding S, Werners FB, Born M, Pallua N, Suschek CV. Effects of blue light irradiation on human dermal fibroblasts. J Photochem Photobiol B 2011;103(2):118-125.

7. Khan I, Arany P. Biophysical approaches for oral wound healing: Emphasis on photobiomodulation. Adv Wound Care 2015;4(12):724-737.

8. Wang Y, Huang YY, Lyu P, Hamblin MR. Photobiomodulation (blue and green light) encourages osteoblastic-differentiation of human adipose-derived stem cells: Role of intracellular calcium and light-gated ion channels. Sci Rep 2016;6:33719.

9. Hamblin MR, Demidova TN. Mechanisms of low level light therapy, in Proceedings of SPIE. 2006;6140:1-12.

10. Terakita A. The opsins. Genome Biol 2005;6(3):213-213.

11. Dartnall HJ, Bowmaker JK, Mollon JD. Human visual pigments: Microspectrophotometric results from the eyes of seven persons. Proc R Soc Lond B Biol Sci 1983; 220(1218):115-130.

12. Kim HJ, Son ED, Jung JY, Choi H, Lee TR, Shin DW. Violet light down-regulates the expression of specific differentiation markers through Rhodopsin in normal human epidermal keratinocytes. PLoS ONE 2013;8(9):1-10.

13. Wicks NL, Chan JW, Najera JA, Ciriello JM, Oancea E. UVA phototransduction drives early melanin synthesis in human melanocytes. Curr Biol 2011;21(22):1906-1911.

14. de Assis LVM, Moraes MN, Magalhaes-Marques KK, Castrucci AML. Melanopsin and rhodopsin mediate UVA-induced immediate pigment darkening: Unravelling the photosensitive system of the skin. Eur J Cell Biol 2018;97(3):150-162.

15. Koyanagi M, Takada E, Nagata T, Tsukamoto H, Terakita A Homologs of vertebrate Opn3 potentially serve as a light sensor in nonphotoreceptive tissue. Proc Natl Acad Sci U S A 2013;110(13):4998-5003.

16. Bailes HJ, Lucas RJ. Human melanopsin forms a pigment maximally sensitive to blue light (lambdamax approximately $479 \mathrm{~nm})$ supporting activation of $\mathrm{G}(\mathrm{q} / 11)$ and $\mathrm{G}(\mathrm{i} / \mathrm{o})$ signalling cascades. Proc Biol Sci 2013;280(1759):20122987.

17. Kojima D, Mori S, Torii M, Wada A, Morishita R, Fukada Y. UV-sensitive photoreceptor protein OPN5 in humans and mice. PLoS ONE 2011;6(10):e26388.

18. Liao X, Xie GH, Liu HW, et al. Helium-neon laser irradiation promotes the proliferation and migration of human epidermal stem cells in vitro: Proposed mechanism for enhanced wound re-epithelialization. Photomed Laser Surg 2014; 32(4):219-225.

19. Haas AF, Isseroff RR, Wheeland RG, Rood PA, Graves PJ. Low-energy helium-neon laser irradiation increases the motility of cultured human keratinocytes. J Invest Dermatol 1990;94(6):822-826

20. Webb C, Dyson M, Lewis WH. Stimulatory effect of $660 \mathrm{~nm}$ low level laser energy on hypertrophic scar-derived fibroblasts: Possible mechanisms for increase in cell counts. Lasers Surg Med 1998;22(5):294-301.

21. Houreld N, Abrahamse H. Low-intensity laser irradiation stimulates wound healing in diabetic wounded fibroblast cells (WS1). Diabetes Technol Ther 2010;12(12):971-978.

22. Taflinski L, Demir E, Kauczok J, et al. Blue light inhibits transforming growth factor-beta1-induced myofibroblast differentiation of human dermal fibroblasts. Exp Dermatol 2014;23(4):240-246.

23. Mignon C, Uzunbajakava NE, Raafs B, Botchkareva NV, Tobin DJ. Photobiomodulation of human dermal fibroblasts in vitro: Decisive role of cell culture conditions and treatment protocols on experimental outcome. Sci Rep 2017;7(1):2797.

24. Masson-Meyers DS, Bumah VV, Enwemeka CS. Blue light does not impair wound healing in vitro. J Photochem Photobiol B 2016;160:53-60.

25. Wang Y, Wang Y, Wang Y, et al. Antimicrobial blue light inactivation of pathogenic microbes: State of the art. Drug Resist Updat 2017;33-35(Supplement C):1-22.

26. Dai T, Gupta A, Huang Y-Y, et al. Blue light rescues mice from potentially fatal Pseudomonas aeruginosa burn infection: Efficacy, safety, and mechanism of action. Antimicrob Agents Chemother 2013;57(3):1238-1245.

27. Pfaff S, Liebmann J, Born M, Merk HF, von Felbert V. Prospective randomized long-term study on the efficacy and safety of UV-free blue light for treating mild psoriasis vulgaris. Dermatology 2015;231(1):24-34.

28. Kleinpenning MM, Otero ME, van Erp PE, Gerritsen MJ, van de Kerkhof PC. Efficacy of blue light vs. red light in the treatment of psoriasis: A double-blind, randomized comparative study. J Eur Acad Dermatol Venereol 2012;26(2):219-225.

29. Garza ZCF, Born M, Hilbers PAJ, van Riel NAW, Liebmann J. Visible light therapy: Molecular mechanisms and therapeutic opportunities. Curr Med Chem 2017. https://doi.org/10.2174/ 0929867324666170727112206

30. Mamalis A, Garcha M, Jagdeo J. Light emitting diodegenerated blue light modulates fibrosis characteristics Fibroblast proliferation, migration speed, and reactive oxygen species generation. Lasers Surg Med 2015;47(2):210-215.

31. Sikka G, Hussmann GP, Pandey D, et al. Melanopsin mediates light-dependent relaxation in blood vessels. Proc Natl Acad Sci U S A 2014;111(50):17977-17982.

32. Miyashita Y, Moriya T, Asami K, Kubota T, Yamada K. Expression of opsin molecule in cultured murine melanocyte. J Investig Dermatol Symp Proc 2001;6(1):54-57. 
33. Buscone S, Mardaryev AN, Raafs B, et al. A new path in defining light parameters for hair growth: Discovery and modulation of photoreceptors in human hair follicle. Lasers Surg Med 2017;49(7):705-718.

34. Toh PP, Bigliardi-Qi M, Yap AM, Sriram G, Bigliardi P. Expression of peropsin in human skin is related to phototransduction of violet light in keratinocytes. Exp Dermatol 2016;25(12):1002-1005.

35. Regazzetti C, Sormani L, Debayle D, et al. Melanocytes sense blue light and regulate pigmentation through the Opsin3. J Invest Dermatol 2017;138(1):171-178.

36. Kleinpenning MM, Smits T, Frunt MH, Van Erp PE, Van De Kerkhof PC, Gerritsen RM. Clinical and histological effects of blue light on normal skin. Photodermatol Photoimmunol Photomed 2010;26(1):16-21.

37. Stojadinovic O, Tomic-Canic M. Human ex vivo wound healing model. Methods Mol Biol 2013;1037:255-264.

38. Kajagar BM, Godhi AS, Pandit A, Khatri S. Efficacy of low level laser therapy on wound healing in patients with chronic diabetic foot ulcers-a randomised control trial. Indian J Surg 2012;74(5):359-363.

39. Lippert J, Halfter H, Heidbreder A, et al. Altered dynamics in the circadian oscillation of clock genes in dermal fibroblasts of patients suffering from idiopathic hypersomnia. PLoS ONE 2014;9(1):e85255.

40. Pomari E, Dalla Valle L, Pertile P, Colombo L, Thornton MJ. Intracrine sex steroid synthesis and signaling in human epidermal keratinocytes and dermal fibroblasts. FASEB J 2015;29(2):508-524.

41. Haltaufderhyde K, Ozdeslik RN, Wicks NL, Najera JA, Oancea E. Opsin expression in human epidermal skin. Photochem Photobiol 2015;91(1):117-123.

42. Janich P, Toufighi K, Solanas G, et al. Human epidermal stem cell function is regulated by circadian oscillations. Cell Stem Cell 2013;13(6):745-753.

43. Tsutsumi M, Ikeyama K, Denda S, et al. Expressions of rod and cone photoreceptor-like proteins in human epidermis. Exp Dermatol 2009;18(6):567-570.

44. Panda S, Sato TK, Castrucci AM, et al. Melanopsin (Opn4) requirement for normal light-induced circadian phase shifting. Science 2002;298(5601):2213-2216.

45. Hankins MW, Peirson SN, Foster RG. Melanopsin: An exciting photopigment. Trends Neurosci 2008;31(1):27-36

46. Poletini MO, Ramos BC, Moraes MN, Castrucci AM. Nonvisual opsins and the regulation of peripheral clocks by light and hormones. Photochem Photobiol 2015; 91(5):1046-1055

47. Do MTH, Yau K-W. Intrinsically photosensitive retinal ganglion cells. Physiol Rev 2010;90(4):1547.

48. Pérez-Cerezales S, Boryshpolets S, Afanzar O, et al. Involvement of opsins in mammalian sperm thermotaxis. Sci Rep $2015 ; 5: 16146$

49. Moraes MN, de Assis LVM, Magalhães-Marques KK, Poletini MO, de Lima LHRG, Castrucci AMdL. Melanopsin, a canonical light receptor, mediates thermal activation of clock genes. Sci Rep 2017;7(1):13977.

50. Leung NY, Montell C. Unconventional roles of opsins. Annu Rev Cell Dev Biol 2017;33(1):241-264.

51. Ebrey T, Koutalos Y. Vertebrate photoreceptors. Prog Retin Eye Res 2001;20(1):49-94.

52. Barreto Ortiz S, Hori D, Nomura Y, et al. Opsin 3 and 4 mediate light-Induced pulmonary vasorelaxation that is potentiated by G-Protein receptor kinase 2 inhibition. Am J Physiol Lung Cell Mol Physiol 2017;314(1):L93-L106.

53. Ondrusova K, Fatehi M, Barr A, et al. Subcutaneous white adipocytes express a light sensitive signaling pathway mediated via a melanopsin/TRPC channel axis. Sci Rep 2017;7(1):16332.

54. Lister T, Wright PA, Chappell PH. Optical properties of human skin. J Biomed Opt 2012;17(9):90901-90901.

55. Yamashita T, Ohuchi H, Tomonari S, Ikeda K, Sakai K, Shichida Y. Opn5 is a UV-sensitive bistable pigment that couples with Gi subtype of G protein. Proc Natl Acad Sci U S A 2010;107(51):22084-22089.

56. Blanpain C, Fuchs E. Epidermal homeostasis: A balancing act of stem cells in the skin. Nat Rev Mol Cell Biol 2009;10(3):207-217.

57. Singh SK, Kurfurst R, Nizard C, Schnebert S, Perrier E, Tobin DJ. Melanin transfer in human skin cells is mediated by filopodia-a model for homotypic and heterotypic lysosomerelated organelle transfer. FASEB J 2010;24(10):3756-3769.

58. Seery JP, Watt FM. Asymmetric stem-cell divisions define the architecture of human oesophageal epithelium. Curr Biol 2000;10(22):1447-1450

59. Besaratinia A, Yoon J-i, Schroeder C, Bradforth SE, Cockburn M, Pfeifer GP. Wavelength dependence of ultraviolet radiation-induced DNA damage as determined by laser irradiation suggests that cyclobutane pyrimidine dimers are the principal DNA lesions produced by terrestrial sunlight. FASEB J 2011;25(9):3079-3091.

60. Eyerich S, Eyerich K, Traidl-Hoffmann C, Biedermann T. Cutaneous barriers and skin immunity: Differentiating a connected network. Trends Immunol 39(4):315-327.

61. Barriere G, Fici P, Gallerani G, Fabbri F, Rigaud M. Epithelial mesenchymal transition: A double-edged sword. Clin Transl Med 2015;4:14.

62. Kumar V, Bouameur JE, Bar J, et al. A keratin scaffold regulates epidermal barrier formation, mitochondrial lipid composition, and activity. J Cell Biol 2015;211(5):1057-1075.

63. Schauber J, Gallo RL. Expanding the roles of antimicrobial peptides in skin: Alarming and arming keratinocytes. J Invest Dermatol 2007;127(3):510-512.

64. Horng HC, Chang WH, Yeh CC, et al. Estrogen effects on wound healing. Int J Mol Sci 2017;18(11):2325.

65. Wolf Horrell E, D'Orazio J. UV-independent induction of beta defensin 3 in neonatal human skin explants. F1000Res 2014;3:288.

66. Plikus MV, Van Spyk EN, Pham K, et al. The circadian clock in skin: Implications for adult stem cells, tissue regeneration, cancer, aging, and immunity. J Biol Rhythms 2015; 30(3):163-182.

67. de Assis LVM, Moraes MN, da Silveira Cruz-Machado S, Castrucci AML. The effect of white light on normal and malignant murine melanocytes: A link between opsins, clock genes, and melanogenesis. Biochim Biophys Acta 2016;1863(6, Part A):1119-1133.

68. Oh PS, Kim HS, Kim EM, et al. Inhibitory effect of blue light emitting diode on migration and invasion of cancer cells. J Cell Physiol 2017;232(12):3444-3453.

69. Syrovatkina V, Alegre KO, Dey R, Huang XY. Regulation, signaling, and physiological functions of G-Proteins. J Mol Biol 2016;428(19):3850-3868.

70. Wikramanayake TC, Stojadinovic O, Tomic-Canic M. Epidermal differentiation in barrier maintenance and wound healing. Adv Wound Care 2014;3(3):272-280. 